\title{
Prevalence and determinants of depressive symptoms in patients with Chagas cardiomyopathy and predominantly preserved cardiac function
}

\author{
Whesley Tanor Silva ${ }^{[1]}$, Matheus Ribeiro Ávila ${ }^{[1]}$, Lucas Frois Fernandes de Oliveira ${ }^{[1]}$, \\ Pedro Henrique Scheidt Figueiredo ${ }^{[1]}$, Vanessa Pereira Lima ${ }^{[1]}$, Alessandra de Carvalho Bastone ${ }^{[1]}$, \\ Fábio Silva Martins da Costa ${ }^{[2], ~ M a u r o ~ F e l i p p e ~ F e l i x ~ M e d i a n o ~}{ }^{[3]}$, Henrique Silveira Costa ${ }^{[1],[2]}$ \\ and Manoel Otávio da Costa Rocha ${ }^{[2]}$
}

\author{
[1]. Universidade Federal dos Vales do Jequitinhonha e Mucuri, Faculdade de Ciências Biológicas e da Saúde, \\ Departamento de Fisioterapia, Diamantina, MG, Brasil. \\ [2]. Universidade Federal de Minas Gerais, Escola de Medicina, \\ Curso de Pós-Graduação em Infectologia e Medicina Tropical, Belo Horizonte, MG, Brasil. \\ [3]. Fundação Oswaldo Cruz, Instituto Nacional de Infectologia Evandro Chagas, Rio de Janeiro, RJ, Brasil.
}

\begin{abstract}
Introduction: Chagas cardiomyopathy $(\mathrm{ChC})$ is highly stigmatized, and the presence of depressive symptoms may be a common feature. However, its determinants remain unclear. Therefore, the present study aimed to verify the prevalence of depression and the clinical, echocardiographic, functional, and quality of life factors associated with depressive symptoms in patients with ChC and predominantly preserved cardiac function. Methods: Thirty-five patients with ChC (aged 40 to 60 years, 66\% men, NYHA I-III) were evaluated by echocardiography, cardiopulmonary exercise testing, 6-minute walk test (6MWT), and Mini-Mental State Examination. Physical activity level was assessed using the Human Activity Profile (HAP) and health-related quality of life was assessed using the Short-Form Health Survey (SF-36). Depressive symptoms were evaluated using the Beck Depression Inventory. A cutoff point greater than 9 was indicative of depression. Results: Depression was detected in 13 patients (37\%). In the univariate analysis, female sex, NYHA functional class, body mass index, HAP score, mental summary of SF-36, peak oxygen uptake, and 6MWT distance were associated with depressive symptoms. The final model showed that only the HAP score $(\mathrm{B}=-0.533 ; 95 \%$ confidence interval $[\mathrm{CI}]$ : -0.804 to -0.262$)$ and SF-36 mental summary $(B=-0.269 ; 95 \%$ CI: -0.386 to -0.153$)$ remained as independent predictors of depressive symptoms in patients with ChC. Conclusions: Depression was prevalent in patients with $\mathrm{ChC}$ and predominantly preserved cardiac function. Physical activity and mental health were independent risk factors for depressive symptoms.
\end{abstract}

Keywords: Chagas disease. Chagas cardiomyopathy. Depressive disorder. Exercise test. Activities of daily living. Mental health.

\section{INTRODUCTION}

Depression is a result of the complex interactions among social, psychological, and biological factors ${ }^{1}$. It is a very prevalent health condition that stands out for being the leading cause of "lost years" due to disability worldwide ${ }^{1,2}$. This becomes even more important when it is associated with another disease ${ }^{1}$, such as some cardiovascular disorders ${ }^{3,4}$, in which the presence of depressive

\footnotetext{
Corresponding author: Whesley Tanor Silva.

e-mail:whesleytanor@gmail.com

(D) https://orcid.org/0000-0002-5783-1820

Received 16 March 2020

Accepted 18 September 2020
}

symptoms may contribute to a worse prognosis related to morbidity, mortality, poor treatment adherence, and functional decline ${ }^{5}$. In the context of cardiovascular disease, Chagas cardiomyopathy $(\mathrm{ChC})$ stands out because individuals with this disease often face social and economic discrimination due to the stigma of poverty and the fear of sudden cardiac death ${ }^{6}$.

Chagas disease is an infection caused by the protozoan Trypanosoma cruzi. The disease is endemic in Latin America, with an increased prevalence in non-endemic countries such as the United States and some regions of Europe due to migration to these areas ${ }^{7,8}$. Among patients with this disease, $30 \%$ to $40 \%$ experience the cardiac form of the infection, which is characterized by constant inflammation, loss of cardiac muscle fibers, systolic 
dysfunction, functional impairment, and worse prognosis than other cardiopathies ${ }^{9,10}$, which in turn may be associated with mental torment and the onset of depressive symptoms ${ }^{3,11}$.

In patients with Chagas disease, most studies and clinicians have focused on the body structure and function components such as cardiac function ${ }^{12,13}$, myocardial dilation ${ }^{14}$, and functional capacity ${ }^{10}$. Despite the importance of the presence of depressive symptoms in Chagas disease and the impact of these symptoms on clinical management, few studies have investigated these symptoms. Forsythy ${ }^{15}$ reported that patients with Chagas disease typically experience emotional stress since the diagnosis of the disease. Ozaki and de Almeida ${ }^{16}$ found that $41.63 \%$ of the patients with Chagas disease presented with depressive symptoms $(n=110$; comprising indeterminate, cardiac, digestive, and mixed forms). Despite the high prevalence, the determinants of depressive symptoms remain unclear, especially in the early stages of heart disease. Early recognition of the determinants of depressive symptoms may aid in clinical management and improve the health-related quality of life of this neglected population.

Therefore, this study aimed to verify the prevalence of depressive symptoms and their clinical, echocardiographic, and functional determinants in patients with $\mathrm{ChC}$ and predominantly preserved cardiac function.

\section{METHODS}

This was a cross-sectional study conducted at the outpatient clinic for Chagas disease in an endemic area in the state of Minas Gerais, Brazil. Clinically stable patients with $\mathrm{ChC}$ were recruited between June 2019 and March 2020. The research was conducted in accordance with the principles of the Declaration of Helsinki ${ }^{17}$ and was approved by the Institutional Ethics Committee (protocol CAAE 16379719.5.0000.5108, 07/22/2019). All the patients were instructed about the objectives and methods of the study and provided written informed consent before participating.

A minimum of two different serological tests (indirect immunofluorescence, enzyme-linked immunosorbent assay, or indirect hemagglutination) for antibodies against Trypanosoma cruzi were required for the diagnosis of Chagas disease. Serological tests were performed simultaneously, and the diagnosis was confirmed when both methods were reactive. To be included in the present study, patients were required to be between 40 and 60 years of age and to have electrocardiographic and/or echocardiographic abnormalities compatible with $\mathrm{ChC}$ according to I Latin American Guideline ${ }^{18}$. Patients with $\mathrm{ChC}$ who presented with electrocardiographic or echocardiographic changes but with normal global ventricular function were classified as stage B1. Patients with $\mathrm{ChC}$ and global ventricular dysfunction but without previous or current signs and symptoms of chronic heart failure were classified as having stage B2. Exclusion criteria were the use of antidepressant medications, the presence of cardiopathy by other causes, cognitive impairment as determined by the Mini Mental State Examination, and inability to perform the procedures.

All patients underwent clinical examination, echocardiography, and cardiopulmonary exercise testing (CPET), followed by the 6-minute walking test (6MWT). The Mini-Mental State
Examination, Beck Depression Inventory (BDI), Human Activity Profile (HAP), and Short-Form Health Survey (SF-36) questionnaires were administered. The researchers responsible for each evaluation were blinded to the results of the other tests.

\section{HAP}

The HAP is a self-reported questionnaire composed of 94 activities that exhibit variable low- to high-energy requirements, in which the order of the activities is based on their estimated metabolic equivalents. Higher scores were associated with higher physical activity levels ${ }^{20}$. The HAP has been used to evaluate physical activity in research and clinical settings for a variety of health conditions, including $\mathrm{ChC}^{19,20}$.

\section{BDI}

The BDI, an inventory already used in patients with $\mathrm{ChC}^{16}$, was used to verify the presence of depressive symptoms. The inventory comprises 21 questions that address the main types of symptoms and attitudes associated with depression, namely, mood, pessimism sense of failure, self-dissatisfaction, guilt, sense of punishment, self-dislike, self-accusations, suicidal ideas, crying, irritability, social withdrawal, indecisiveness, distortion of body self-image, work difficulty, insomnia, fatigability, appetite, weight loss, somatic occupation, and loss of libido. The patient evaluated the intensity of the symptoms on a scale of 1 to 3, according to Gorenstein ${ }^{21}$. According to the BDI score, the patients were classified into the following levels of depression: no depression or minimal depression $=0-9$, mild to moderate depression $=10-18$, moderate to severe depression $=19-29$, and severe depression $=30-63$, as validated for the Brazilian population ${ }^{22}$.

\section{CPET}

CPET is the gold standard in functional evaluation ${ }^{23}$ and in the present study, it was performed on a treadmill with the metabolic analysis system MetaLyzer 3B (Cortex Medical, Leipzig, Germany). The peak VO2 and minute ventilation-carbon dioxide production (VE/VCO2) slope were used as the primary endpoints in the functional evaluation and were obtained according to current guidelines ${ }^{24}$.

\section{Echocardiographic Evaluation}

An echocardiogram was performed to verify the left ventricular ejection fraction (LVEF) and left ventricular end-diastolic diameter (LVDd), according to the American Society of Echocardiography ${ }^{25}$. The LVEF was obtained using the modified Simpson rule.

\section{Mini-Mental State Examination}

The Mini-Mental State Examination was used to assess the patients' cognitive functions. It is a questionnaire composed of 20 questions related to domains such as attention, language, word memory, and orientation in time and space, and is used by clinicians and researchers to screen for cognitive impairment. The final score varied from 0 to 30, and a result of less than or equal to 23 was considered to indicate cognitive impairment ${ }^{26,27}$.

\section{SF-36 Questionnaire}

SF-36 was used to assess health-related quality of life (HRQoL). This questionnaire consists of eight domains addressing physical 
(functional capacity, physical aspects, pain, vitality, and general health) and mental (social aspects, and emotional and mental health) aspects, and the final score varied from 0 (the most affected) to 100 (without commitment). The questionnaire has been previously translated to Brazilian Portuguese ${ }^{28}$. In the present study, the answers of the physical and mental components were summarized.

\section{MWT}

The 6MWT is a field test widely used for the functional evaluation of patients with $\mathrm{ChC}^{29-31}$. The test was performed according to American Thoracic Society guidelines ${ }^{32}$, and the volunteers were instructed to walk at the highest possible speed without running for over a distance of $30 \mathrm{~m}$ in a period of $6 \mathrm{~min}$. Standardized words of encouragement were provided every minute, and the test was applied twice with a 15-min rest interval. The target variable was the longest distance covered by the patient.

\section{Statistical Analysis}

Statistical analyses were performed using Statistical Package for the Social Sciences (SPSS), version 17.0. Normal distribution of the data was verified using the Kolmogorov-Smirnov test. Continuous data were expressed as mean and 95\% confidence interval (CI) and categorical variables as absolute number and percentage. A univariate regression analysis was performed to verify the association between the BDI score and demographic, clinical, echocardiographic, and functional variables. A multivariate regression analysis was performed to determine the independent predictors of depressive symptoms; those variables with a significance level less than $10 \%(p<0.1)$ were included in the final model.

\section{RESULTS}

Fifty-nine patients were recruited for the present study. Out of these, 14 patients were excluded because they had non-cardiac forms of Chagas disease, six were using antidepressant medications, two had cognitive impairment, one had significant mitral stenosis associated with Chagas disease, and one was not able to perform the exercise test due to advanced knee arthrosis. Thus, 35 patients with $\mathrm{ChC}$ fulfilled the inclusion criteria and were evaluated. The average score found after administration of the BDI was 9 (95\% CI: 6-12). In the sample, 13 (37\%) patients had depression. Among these, $62 \%$ had mild-to-moderate depression and 38\% had moderate-tosevere depression. None of the participants presented with severe depression. The average left ventricular ejection fraction was 54 (95\% CI: 48-59), which characterized preserved cardiac function. The clinical, demographic, echocardiographic, and functional characteristics of the patients are shown in Table 1.

When the sample was stratified, the group of patients with depression had a higher body mass index, lower physical activity level, and lower score in the mental component of the SF-36 when compared to the patients without depression. No differences were observed in the clinical, echocardiographic, and exercise tests. The differences between the groups are shown in Table 2.

In the univariate analysis, female sex, NYHA functional class, body mass index, HAP score, mental summary of SF-36, peak oxygen uptake, and 6MWT distance were associated with the
TABLE 1: Characteristics of the participants included in the study $(n=35)$.

\begin{tabular}{lc}
\hline Variable & Mean $(\mathbf{9 5} \% \mathbf{C l}), \mathbf{n}(\%)$ \\
\hline Age (years) & $48(44-51)$ \\
Male sex (n) & $23(66)$ \\
NYHA functional class & \\
I & $20(57)$ \\
II & $9(26)$ \\
III & $6(17)$ \\
Clinical classification & \\
Stage B1 & $20(57)$ \\
Stage B2 & $15(43)$ \\
BMI (kg/m²) & $27(25-28)$ \\
SBP (mmHg) & $102(98-107)$ \\
DBP (mmHg) & $67(64-69)$ \\
HR (bpm) & $67(63-70)$ \\
HAP (score) & $84(81-86)$ \\
SF-36 physical summary (score) & $48(45-52)$ \\
SF-36 mental summary (score) & $48(42-55)$ \\
Mini-Mental State Examination (score) & $24(22-25)$ \\
VO2peak (ml.kg.min) & $26(23-29)$ \\
VE/VCO2 slope & $32(30-33)$ \\
6MWT (m) & $548(518-578)$ \\
LVEF (\%) & $54(48-59)$ \\
LVDd (mm) & $55(51-59)$ \\
\hline
\end{tabular}

Data are presented as mean and $95 \%$. Cl: confidence interval. BMI: body mass index; SBP: systolic blood pressure; DBP: diastolic blood pressure; HR: heart rate; HAP: Human Profile Activity; VO2peak: peak oxygen uptake; VE/VCO2 slope: minute ventilation/carbon dioxide production relationship; 6MWT: six-minute walk test; LVEF: left ventricular ejection fraction; LVDd: left ventricular end-diastolic diameter.

BDI score. In the multivariate model, only the HAP score and the mental summary of SF-36 remained as independent predictors of the BDI score in patients with ChC. The adjusted $\mathrm{r}^{2}$ of the model, including the two variables, was 0.66 . The results of the univariate and multivariate analyses are shown in Table 3.

\section{DISCUSSION}

To the best of our knowledge, this is the first study to investigate the clinical, echocardiographic, functional, and quality of life factors associated with depressive symptoms in patients with $\mathrm{ChC}$. Our results demonstrated that: (1) depressive symptoms were prevalent in the study population, with a rate of $37 \%$, (2) physical activity level, assessed by the HAP score, and mental health, assessed by the mental summary of SF-36, were strongly related to depressive symptoms in patients with $\mathrm{ChC}$.

Depressed patients have decreased life expectancies, and cardiovascular disease may be one possible explanation for the increased risk of premature death in these patient ${ }^{33}$. In the context of Chagas disease, a qualitative study reported that the initial discussions among respondents were regarding their experiences or fears about the disease and the possibility of death ${ }^{34}$. The disease was characterized as a precursor to high depression levels, possibly because of the risk of sudden death related to the cardiac form ${ }^{35}$. In the present study, depressive symptoms were found in $37 \%$ of patients with ChC. Ozaki et al. ${ }^{16}$ verified that in a sample comprising patients with different clinical forms of the disease, the prevalence 
Silva WT et al. - Depressive symptoms in Chagas cardiomyopathy

TABLE 2: Demographic, clinical, echocardiographic, functional, and quality of life variables stratified by the categories of depressive symptoms.

\begin{tabular}{|c|c|c|c|}
\hline Variables & $\begin{array}{l}\text { With depressive } \\
\text { symptoms }(n=13)\end{array}$ & $\begin{array}{l}\text { Without depressive } \\
\text { symptoms }(n=22)\end{array}$ & p-value \\
\hline Age (years) & $49(44-54)$ & $47(43-51)$ & 0.753 \\
\hline Male sex (n) & $7(54)$ & $16(73)$ & 0.220 \\
\hline \multicolumn{4}{|l|}{ NYHA functional class } \\
\hline 1 & $7(54)$ & $13(59)$ & \multirow{3}{*}{0.208} \\
\hline II & $2(15)$ & $7(32)$ & \\
\hline III & $4(31)$ & $2(9)$ & \\
\hline BMI $\left(\mathrm{kg} / \mathrm{m}^{2}\right)$ & $28(26-31)$ & $26(23-28)$ & 0.042 \\
\hline $\mathrm{SBP}(\mathrm{mmHg})$ & $100(94-106)$ & $104(97-111)$ & 0.693 \\
\hline $\mathrm{DBP}(\mathrm{mmHg})$ & $67(63-72)$ & $66(63-70)$ & 0.490 \\
\hline HR (bpm) & $64(58-70)$ & $69(64-73)$ & 0.135 \\
\hline HAP (score) & $79(76-82)$ & $86(83-90)$ & 0.002 \\
\hline SF-36 physical summary (score) & $48(42-55)$ & $48(44-53)$ & 0.662 \\
\hline SF-36 mental summary (score) & $31(22-40)$ & $58(54-62)$ & $<0.001$ \\
\hline Mini-Mental State Examination (score) & $23(21-25)$ & $24(22-26)$ & 0.184 \\
\hline VO2peak (ml.kg.min) & $24(18-30)$ & $28(24-31)$ & 0.491 \\
\hline VE/VCO2 slope & $34(31-36)$ & $31(29-33)$ & 0.390 \\
\hline 6MWT (m) & $533(475-591)$ & $557(520-595)$ & 0.689 \\
\hline LVEF (\%) & $51(40-63)$ & $55(49-61)$ & 0.608 \\
\hline LVDd (mm) & $57(48-66)$ & $54(49-59)$ & 0.701 \\
\hline
\end{tabular}

Values highlighted in bold were statistically significant $(\mathrm{p}$-value $<0.05)$.

TABLE 3: Univariate and multivariate predictors of BDI scores in patients with $\mathrm{ChC}$.

\begin{tabular}{|c|c|c|c|c|c|c|c|}
\hline \multirow{2}{*}{ Variables } & \multicolumn{4}{|c|}{ Univariate analysis } & \multicolumn{3}{|c|}{ Multivariate analysis } \\
\hline & $\mathbf{R}$ & Beta-coefficient & $95 \% \mathrm{Cl}$ & p-value & Beta-coefficient & $95 \% \mathrm{Cl}$ & $p$-value \\
\hline Constant & - & - & - & - & 66.214 & 44.689 to 87.739 & $<0.001$ \\
\hline Age & 0.137 & 0.13 & -0.23 to 0.50 & 0.431 & - & - & - \\
\hline Female sex & 0.399 & 6.60 & 12.00 to 1.22 & 0.018 & -2.222 & -6.685 to 2.242 & 0.313 \\
\hline NYHA class & 0.411 & 4.23 & 0.91 to 7.56 & 0.014 & 0.064 & -4.535 to 4.663 & 0.977 \\
\hline BMI & 0.440 & 0.79 & 0.21 to 1.37 & 0.009 & 0.177 & -0.324 to 0.678 & 0.472 \\
\hline SBP & 0.070 & 0.04 & -0.18 to 0.27 & 0.690 & - & - & - \\
\hline DBP & 0.223 & 0.25 & -0.13 to 0.62 & 0.197 & - & - & - \\
\hline HR & 0.208 & -0.17 & -0.47 to 0.12 & 0.231 & - & - & - \\
\hline HAP & 0.631 & -0.72 & -1.06 to -0.39 & $<0.001$ & -0.533 & -0.804 to -0.262 & $<0.001$ \\
\hline SF-36 physical summary & 0.074 & -0.07 & -0.41 to 0.27 & 0.682 & - & - & - \\
\hline SF-36 mental summary & 0.694 & -0.35 & -0.48 to -0.22 & $<0.001$ & -0.269 & -0.386 to -0.153 & $<0.001$ \\
\hline Mini-Mental State Examination & 0.283 & -0.50 & -1.11 to 0.11 & 0.105 & - & - & - \\
\hline VO2peak & 0.313 & -0.31 & -0.65 to 0.03 & 0.071 & 0.177 & -0.505 to 0.450 & 0.907 \\
\hline VE/VCO2 slope & 0.217 & -0.41 & -0.24 to 1.06 & 0.211 & - & - & - \\
\hline 6MWT & 0.315 & -0.03 & -0.06 to 0.01 & 0.066 & -0.016 & -0.048 to 0.015 & 0.293 \\
\hline LVEF & 0.125 & -0.07 & -0.26 to 0.12 & 0.481 & - & - & - \\
\hline LVDd & 0.121 & 0.08 & -0.16 to 0.33 & 0.489 & - & - & - \\
\hline
\end{tabular}

Variables in bold in the univariate analysis $(p$-value $<0.10$ ) were included in the multivariate linear regression model. 
of depressive symptoms was approximately $41 \%$. The findings of both the studies were similar and highlighted the high prevalence of such symptoms in this population.

We also found that female sex, body mass index, functional performance, physical status, and mental health were associated with depressive symptoms. Previous studies ${ }^{36,37}$ have already shown a higher prevalence of depressive symptoms in women with $\mathrm{ChC}$ when compared to men, which has also been observed in other populations $^{38,39}$. Sex differences in biological, psychological, and environmental factors, as well as many hormonal changes caused by the menstrual cycle, pregnancy, postpartum period, abortion, and menopause may explain the association of the female sex with depressive symptoms ${ }^{40}$. The body mass index is closely linked to self-esteem. Functional status was strongly associated with depressive symptoms in cardiac patients, and it can be a valuable tool in identifying patients at risk of hospitalization ${ }^{41}$. However, in the final multivariate model, we found that only mental health and the physical activity level remained independent predictors of the BDI score.

The association between mental health and depressive symptoms can be explained by several factors such as fear of death, difficulty in accessing appropriate treatment, and mental stress emerging immediately after diagnosis ${ }^{15}$. In addition, Chagas disease is considered to be one of the most neglected tropical diseases. It is often associated with poverty and characterized by stigma, which in turn can be a psychological and social burden, leading to progressive social exclusion, reduced health-related quality of life, and mental disorders ${ }^{42}$. In a meta-analysis, Daré et al ${ }^{43}$ demonstrated that the association between parasitic diseases (including Chagas disease) and mental disorders (anxiety, depression, bipolar disorder, and schizophrenia) were relatively high in developing and emerging countries. Thus, stigma must be considered an important component in the emergence of depressive symptoms, as it leads to despair, hopelessness, embarrassment, increased stress, and depression ${ }^{44}$. The concern about mental health in patients with $\mathrm{ChC}$ has grown in recent years. A recent longitudinal study ${ }^{45}$ showed that the mental component of SF-36 was an independent predictor of adverse cardiovascular events in these patients. This finding, together with the results of the present study, reinforces the need for clinical and psychological management of the mental health of patients with $\mathrm{ChC}$.

The inverse association between physical activity and depressive symptoms is well established in the literature ${ }^{46}$. A population-based study with 26,615 middle-aged and older healthy participants at baseline investigated the associations of estimated cardiorespiratory fitness with depression and reported that a medium and high level of cardiorespiratory fitness was cross-sectionally and prospectively associated with lower odds of depression ${ }^{47}$. Moreover, physical inactivity has been shown to partially mediate the relationship between depression and mortality among persons with cardiovascular disease ${ }^{48,49}$. Results from the Cardiovascular Health Study, a multicenter prospective cohort study with community-dwelling older adults, showed that depressive symptoms and physical inactivity independently increased the risk of cardiovascular mortality and were strongly also associated with each other. In addition, individuals with both the conditions had a greater risk of cardiovascular mortality than those with only one condition ${ }^{50}$. In our study, patients with depression presented with a lower level of physical activity, and physical activity was found to be an independent predictor of depressive symptoms. Considering the effectiveness of exercise training in patients with $\mathrm{ChC}^{51-53}$ and taking into account the relationship between physical inactivity, depressive symptoms, and adverse health events related to cardiovascular disease, physical exercise programs should be considered for this population.

The present study had several strengths and limitations. As a limitation, our sample size was relatively small. In addition, our sample was composed predominantly of patients with preserved cardiac function, and the results should be limited to patients with this disease characteristic. As a strength, the present study demonstrated the association of physical activity and mental health with depressive symptoms in patients with $\mathrm{ChC}$ for the first time.

In conclusion, depression is a prevalent condition in patients with $\mathrm{ChC}$ and predominantly preserved cardiac function. The physical activity level and mental health were found to be independent predictors of depressive symptoms in this population. Therefore, early interventions targeting these modifiable risk factors should be encouraged.

\section{AUTHORS' CONTRIBUTION}

WTS: Conception and design of the study, acquisition of data, wrote the paper. MRA: Acquisition of data, wrote the paper. LFFO: Acquisition of data, wrote the paper. PHSF: Analysis and interpretation of data, critical review of the manuscript. VPL: Analysis and interpretation of data, critical review of the manuscript. ACB: Analysis and interpretation of data, critical review of the manuscript. FSMC: Analysis and interpretation of data. MFFM: Conception and design of the study, critical review of the manuscript. HSC: Conception and design of the study, wrote the paper. MOCR: Conception and design of the study, critical review of the manuscript.

\section{CONFLICT OF INTEREST}

The authors declare that there is no conflict of interest.

\section{REFERENCES}

1. World Health Organization (WHO). Depression. Geneva: WHO; 2019.

2. Smith K. Mental health: a world ofdepression. Nature. 2014;515(7526):181.

3. Cirelli MA, Lacerda MS, Lopes CT, de Lima Lopes J, de Barros A. Correlations between stress, anxiety and depression and sociodemographic and clinical characteristics among outpatients with heart failure. Arch Psychiatr Nurs. 2018;32(2):235-41.

4. Pedersen SS, von Kanel R, Tully PJ, Denollet J. Psychosocial perspectives in cardiovascular disease. Eur J Prev Cardiol. 2017;24(3_suppl):108-15.

5. Suzuki T, Shiga T, Kuwahara K, Kobayashi S, Suzuki S, Nishimura $\mathrm{K}$, et al. Impact of clustered depression and anxiety on mortality and rehospitalization in patients with heart failure. J Cardiol. 2014;64(6):456-62.

6. Dias JCP. Globalização, iniqüidade e doença de Chagas. Cad Saude Publica. 2007;23:S13-S22.

7. Requena-Mendez A, Aldasoro E, de Lazzari E, Sicuri E, Brown M, Moore DA, et al. Prevalence of Chagas disease in Latin-American migrants living in Europe: a systematic review and meta-analysis. PLoS Negl Trop Dis. 2015;9(2):e0003540. 
8. Bern C, Kjos S, Yabsley MJ, Montgomery SP. Trypanosoma cruzi and Chagas' Disease in the United States. Clin Microbiol Rev. 2011;24(4):655-81.

9. Perez-Molina JA, Molina I. Chagas disease. Lancet. 2018;391(10115):82-94.

10. Costa HS, Lima MMO, Costa F, Chaves AT, Nunes MCP, Figueiredo PHS, et al. Reduced functional capacity in patients with Chagas disease: a systematic review with meta-analysis. Rev Soc Bras Med Trop. 2018;51(4):421-6.

11. Suman AC, Costa E, Bazan SGZ, Hueb JC, Carvalho FC, Martin LC, et al. Evaluating respiratory musculature, quality of life, anxiety, and depression among patients with indeterminate chronic Chagas disease and symptoms of pulmonary hypertension. Rev Soc Bras Med Trop. 2017;50(2):194-8.

12. Moreira HT, Volpe GJ, Marin-Neto JA, Ambale-Venkatesh B, Nwabuo $\mathrm{CC}$, Trad HS, et al. Evaluation of Right Ventricular Systolic Function in Chagas Disease Using Cardiac Magnetic Resonance Imaging. Circ Cardiovasc Imaging. 2017;10(3):e005571.

13. Tundo F, Lombardi F, Rocha MC, Botoni F, Schmidt G, Barros VC, et al. Heart rate turbulence and left ventricular ejection fraction in Chagas disease. EP Europace. 2005;7(3):197-203.

14. Rassi Ddo C, Vieira ML, Arruda AL, Hotta VT, Furtado RG, Rassi DT, et al. Echocardiographic parameters and survival in Chagas heart disease with severe systolic dysfunction. Arq Bras Cardiol. 2014;102(3):245-52.

15. Forsyth CJ. "I Cannot Be Worried": Living with Chagas Disease in Tropical Bolivia. PLoS Neg1 Trop Dis. 2017;11(1):e0005251.

16. Ozaki Y, Guariento ME, de Almeida EA. Quality of life and depressive symptoms in Chagas disease patients. Qual Life Res. 2011;20(1):133-8.

17. World Medical Association. World Medical Association Declaration of Helsinki: ethical principles for medical research involving human subjects. JAMA. 2013;310(20):2191-4.

18. Andrade JPd, Marin Neto JA, De Paola AAV, Vilas-Boas F, Oliveira GMM, Bacal F, et al. I Latin American Guidelines for the diagnosis and treatment of Chagas' heart disease: executive summary. Arq Bras Cardiol. 2011;96(6):434-42.

19. Davidson M, de Morton N. A systematic review of the Human Activity Profile. Clin Rehabil. 2007;21(2):151-62.

20. Costa HS, Lima MMO, Vieira CFD, Silva WT, Nunes M, Rocha MOC, et al. Assessment of functional performance in Chagas heart disease by Human Activity Profile questionnaire. Disabil Rehabil. 2019:1-5.

21. Gorenstein C, Andrade LHSG. Validation of a Portuguese version of the Beck Depression Inventory and State-Trait anxiety inventory in Brazilian subjects. Braz J Med Biol Res. 1996;29(4):453-7.

22. Cunha J. Brazilian Manual for Beck Scale. 1th ed. Sao Paulo, SP: Casa do Psicologo. 2001. 171p.

23. American Thoracic Society. ATS/ACCP Statement on cardiopulmonary exercise testing. Am J Respir Crit Care Med. 2003;167(2):211

24. Guazzi M, Arena R, Halle M, Piepoli MF, Myers J, Lavie CJ. 2016 Focused Update: Clinical Recommendations for Cardiopulmonary Exercise Testing Data Assessment in Specific Patient Populations. Circulation. 2016;133(24):e694-711.

25. Lang RM, Badano LP, Mor-Avi V, Afilalo J, Armstrong A, Ernande $\mathrm{L}$, et al. Recommendations for cardiac chamber quantification by echocardiography in adults: an update from the American Society of Echocardiography and the European Association of Cardiovascular Imaging. J Am Soc Echocardiogr. 2015;28(1):1-39.e14.

26. Folstein M. A practical method for grading the cognitive state of patients for the children. J Psychiatr Res. 1975;12:189-98.
27. Santiago-Bravo G, Sudo FK, Assunção N, Drummond C, Mattos P. Dementia screening in Brazil: a systematic review of normative data for the mini-mental state examination. Clinics. 2019;74.

28. Ciconelli RM. Tradução para o português e validação do questionário genérico de avaliação de qualidade de vida Medical Outcomes Study 36-Item Short-Form Health Survey (SF-36). Rev Bras Reumatol. 1997;39:143-50

29. Costa HS, Lima MMO, de Sousa GR, de Souza AC, Alencar MCN, Nunes MCP, et al. Functional capacity and risk stratification by the Six-minute Walk Test in Chagas heart disease: Comparison with Cardiopulmonary Exercise Testing. Int J Cardiol. 2014;177(2):661-3.

30. Costa HS, Lima MM, Alencar MC, Sousa GR, Figueiredo PH, Nunes $\mathrm{MC}$, et al. Prediction of peak oxygen uptake in patients with Chagas heart disease: Value of the Six-minute Walk Test. Int J Cardiol. 2017;228:385-7.

31. Costa HS, Lima MMO, Lage SM, da Costa FSM, Figueiredo PHS, da Costa Rocha MO. Six-minute walk test and incremental shuttle walk test in the evaluation of functional capacity in Chagas heart disease. J Exerc Rehabil. 2018;14(5):844-50.

32. [No authors listed]. ATS statement: guidelines for the six-minute walk test. Am J Respir Crit Care Med. 2002;166(1):111-7.

33. Paz-Filho G, Licinio J, Wong M-L. Pathophysiological basis of cardiovascular disease and depression: a chicken-and-egg dilemma. J Exerc Braz J Psychiatry. 2010;32(2):181-91.

34. Sanmartino M, Saavedra AA, Prat JGi, Barba MCP, Albajar-Viñas P. Que no tengan miedo de nosotros: el Chagas según los propios protagonistas. Interface. 2015;19(55):1063-75.

35. Cavalcanti MAF, Nascimento EGCd, Alchieri JC, Andrade CdM. Manifestações e estratégias de enfrentamento da Doença de Chagas que interferem na qualidade de vida do indivíduo: uma revisão sistemática. Cien Saude Colet. 2019;24(4):1405-16.

36. Santos-Filho JC, Vieira MC, Xavier IG, Maciel ER, Rodrigues Junior LF, Curvo EO, et al. Quality of life and associated factors in patients with chronic Chagas disease. Trop Med Int Health. 2018;23(11):1213-22.

37. Oliveira BG, Abreu MNS, Abreu CDG, Rocha MODC, Ribeiro AL. Health-related quality of life in patients with Chagas disease. Rev Soc Bras Med Trop. 2011;44(2):150-6.

38. Linden W, Vodermaier A, Mackenzie R, Greig D. Anxiety and depression after cancer diagnosis: prevalence rates by cancer type, gender, and age. J Affect Disord. 2012;141(2-3):343-51.

39. Angst J, Gamma A, Gastpar M, Lépine J-P, Mendlewicz J, Tylee A. Gender differences in depression. Eur Arch Psychiatry Clin Neurosci. 2002;252(5):201-9.

40. Kuehner C. Why is depression more common among women than among men? Lancet Psychiatry. 2017;4(2):146-58.

41. Schopfer DW, Regan M, Heidenreich PA, Whooley MA. Depressive Symptoms, Cardiac Disease Severity, and Functional Status in Patients With Coronary Artery Disease (from the Heart and Soul Study). Am J Cardio. 2016;118(9):1287-92.

42. Hofstraat K, van Brakel WH. Social stigma towards neglected tropical diseases: a systematic review. Int Health. 2016;8(suppl_1):i53-i70.

43. Daré LO, Bruand P-E, Gérard D, Marin B, Lameyre V, Boumédiène $\mathrm{F}$, et al. Associations of mental disorders and neurotropic parasitic diseases: a meta-analysis in developing and emerging countries. BMC Public Health. 2019;19(1):1645.

44. Litt E, Baker MC, Molyneux D. Neglected tropical diseases and mental health: a perspective on comorbidity. Trends Parasitol. 2012;28(5): 195-201 
45. Costa HS, Lima MMO, Figueiredo PHS, Chaves AT, Nunes MCP, da Costa Rocha MO. The prognostic value of health-related quality of life in patients with Chagas heart disease. Qual Life Res. 2019;28(1):67-72.

46. Brown WJ, Ford JH, Burton NW, Marshall AL, Dobson AJ. Prospective study of physical activity and depressive symptoms in middle-aged women. Am J Prev Med. 2005;29(4):265-72.

47. Shigdel R, Stubbs B, Sui X, Ernstsen L. Cross-sectional and longitudinal association of non-exercise estimated cardiorespiratory fitness with depression and anxiety in the general population: The HUNT study. J Affect Disord. 2019;252:122-9.

48. Whooley MA, De Jonge P, Vittinghoff E, Otte C, Moos R, Carney $\mathrm{RM}$, et al. Depressive symptoms, health behaviors, and risk of cardiovascular events in patients with coronary heart disease. JAMA. 2008;300(20):2379-88.

49. Blumenthal JA, Babyak MA, Carney RM, Huber M, Saab PG, Burg MM, et al. Exercise, depression, and mortality after myocardial infarction in the ENRICHD trial. Med Sci Sports Exerc. 2004;36(5):746-55.
50. Win S, Parakh K, Eze-Nliam CM, Gottdiener JS, Kop WJ, Ziegelstein RC. Depressive symptoms, physical inactivity and risk of cardiovascular mortality in older adults: the Cardiovascular Health Study. Heart. 2011;97(6):500-5.

51. Lima MM, Rocha MO, Nunes MC, Sousa L, Costa HS, Alencar MC, et al. A randomized trial of the effects of exercise training in Chagas cardiomyopathy. Eur J Heart Fail. 2010;12(8):866-73.

52. Mediano MFF, Mendes FdSNS, Pinto VLM, Silva GMSd, Silva PSd, Carneiro FM, et al. Cardiac rehabilitation program in patients with Chagas heart failure: a single-arm pilot study. Rev Soc Bras Med Trop. 2016;49(3):319-28.

53. Fialho PH, Tura BR, Sousa ASd, Oliveira CRd, Soares CCS, Oliveira JRd, et al. Effects of an exercise program on the functional capacity of patients with chronic Chagas' heart disease, evaluated by cardiopulmonary testing. Rev Soc Bras Med Trop. 2012;45(2): $220-4$. 